\title{
Ipsilateral Actions of Feline Corticospinal Tract Neurons on Limb Motoneurons
}

\author{
S. A. Edgley, ${ }^{2}$ E. Jankowska, ${ }^{1}$ and I. Hammar ${ }^{1}$ \\ ${ }^{1}$ Department of Physiology, Göteborg University, 40530 Göteborg, Sweden, and ²Department of Anatomy, Cambridge University CB2 3DY, \\ United Kingdom
}

Contralateral pyramidal tract (PT) neurons arising in the primary motor cortex are the major route through which volitional limb movements are controlled. However, the contralateral hemiparesis that follows PT neuron injury on one side may be counteracted by ipsilateral of actions of PT neurons from the undamaged side. To investigate the spinal relays through which PT neurons may influence ipsilateral motoneurons, we analyzed the synaptic actions evoked by stimulation of the ipsilateral pyramid on hindlimb motoneurons after transecting the descending fibers of the contralateral PT at a low thoracic level. The results show that ipsilateral PT neurons can affect limb motoneurons trisynaptically by activating contralaterally descending reticulospinal neurons, which in turn activate spinal commissural interneurons that project back across to motoneurons ipsilateral to the stimulated pyramidal tract. Stimulation of the pyramids alone did not evoke synaptic actions in motoneurons but potently facilitated disynaptic EPSPs and IPSPs evoked by stimulation of reticulospinal tract fibers in the medial longitudinal fascicle. In parallel with this double-crossed pathway, corticospinal neurons could also evoke ipsilateral actions via ipsilateral descending reticulospinal tract fibers, acting through ipsilaterally located spinal interneurons. Because the actions mediated by commissural interneurons were found to be stronger than those of ipsilateral premotor interneurons, the study leads to the conclusion that ipsilateral actions of corticospinal neurons via commissural interneurons may provide a better opportunity for recovery of function in hemiparesis produced by corticospinal tract injury.

Key words: pyramidal tract; motor system; spinal cord; reticular formation; commissural interneurons; cat

\section{Introduction}

Damage to the corticospinal tract has very severe consequences for voluntary movements. In humans, such damage is followed by a loss of skilled movements with weakness or paralysis (hemiparesis) on the contralateral side (e.g., after stroke). This has been attributed to the fact that crossed descending corticospinal fibers greatly outnumber the uncrossed ones and that only the contralaterally projecting fibers form direct synaptic contacts with primate limb motoneurons (Porter and Lemon, 1993). However, the capability of the small proportion of ipsilaterally descending corticospinal tract neurons (Lacroix et al., 2004) or neurons in ipsilateral cortical motor areas to contribute to the recovery has often been postulated, for instance, by Darian-Smith et al. (1996), Chen et al. (1997), Feydy et al. (2002), and Strens et al. (2003).

In humans, it has been difficult to ascertain which neuronal systems might mediate ipsilateral actions of the motor cortex. However, in animals, some possibilities ranging from transcallosal and subcortical brainstem interconnections to actions mediated at a spinal level have been tested experimentally. In particular, Lawrence and Kuypers (1968) showed that after selective

Received May 19, 2004; revised June 25, 2004; accepted July 13, 2004.

This study was supported by National Institutes of Health Grant NS 40 863. We thank Rauni Larsson for her invaluable assistance.

Correspondence should be addressed to E. Jankowska, Department of Physiology, Medicinaregatan 11, Box 432, 40530 Göteborg, Sweden. E-mail: Elzbieta.Jankowska@physiol.gu.se.

DOI:10.1523/JNEUROSCI.1941-04.2004

Copyright $\odot 2004$ Society for Neuroscience $\quad$ 0270-6474/04/247804-10\$15.00/0 bilateral lesions of the primate pyramidal tracts in the medulla, other descending pathways permit considerable general recovery of reaching and grasping but not independent movements of the digits.

The possibility that reticulospinal (RS) neurons, which are major subcortical relays of corticospinal fibers (Kuypers and Lawrence, 1967; Keizer and Kuypers, 1984, 1989; Matsuyama and Drew, 1997), are important for recovery of function was demonstrated in cats for actions on neck motoneurons (Alstermark et al., 1983, 1992). However, it remained unknown which spinal neurons are involved in ipsilateral actions of corticospinal tract fibers, and the present study focused on interneurons mediating ipsilateral corticospinal actions evoked via reticulospinal neurons.

Previous work has shown a disynaptic coupling between some reticulospinal neurons and contralateral motoneurons via lamina VIII commissural interneurons (Bannatyne et al., 2003; Jankowska et al., 2003; Hammar et al., 2004). Our first question was whether corticofugal fibers excite those reticulospinal neurons, which excite commissural interneurons, which in turn evoke excitation or inhibition of motoneurons on the side of origin of the corticofugal fibers. Pathways that might permit this are indicated in Figure $1 \mathrm{~A}$.

Disynaptic coupling between reticulospinal neurons and ipsilateral motoneurons via interneurons located at the same side as motoneurons has been long known (Grillner et al., 1968; Wilson and Yoshida, 1969; Takakusaki et al., 1989; Floeter et al., 1993; 

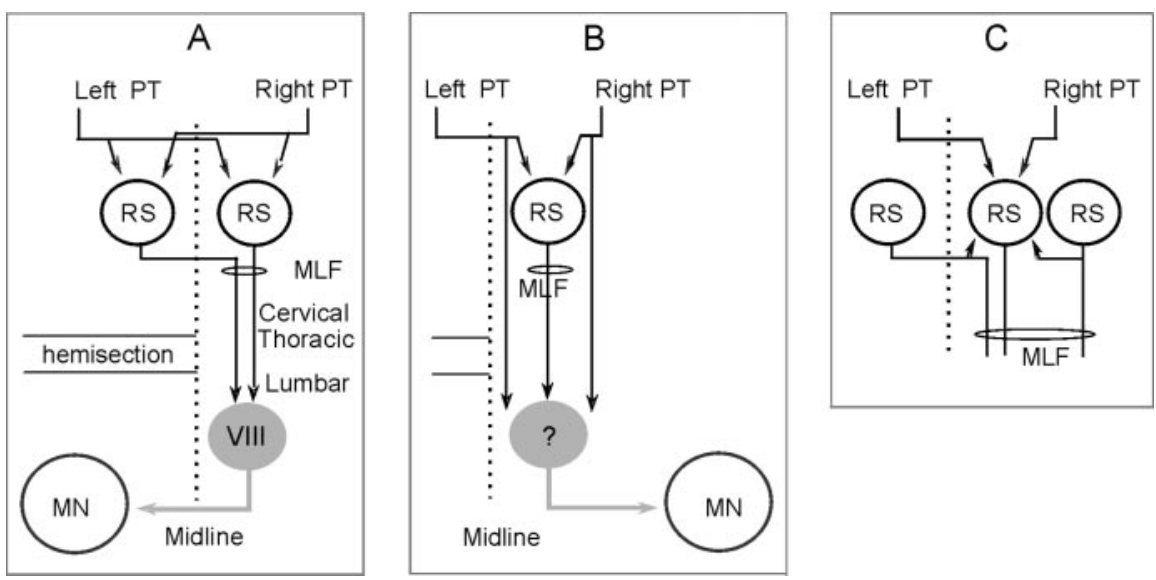

Figure 1. Diagrams showing hypothetical connections between PT fibers and ipsilateral hindlimb motoneurons (MN). $A_{t}$ Connections between left and right pyramidal tract fibers and motoneurons on the left side via left and right $R S$ neurons with axons in the MLF and lumbar commissural interneurons. In preparations in which the left half of the spinal cord was transected at a low thoracic level, ipsilateral actions of left PT fibers on motoneurons could be mediated by RS neurons with axons descending in the right MLF. The direct corticospinal projection is omitted for the sake of simplicity. $B$ is the same as $A$ but for connections with motoneurons on the right side. Ipsilateral actions of right PT fibers on motoneurons are mediated by RS neurons with axons descending in the right MLF and local ipsilateral premotor interneurons indicated by a question mark. Intact corticospinal tract fibers on the right side of the spinal cord are added, but RS neurons located on the left side are omitted for the sake of simplicity. C, A diagram explaining facilitation of synaptic actions evoked from MLF actions by preceding PT stimuli, considering that PT fibers coexcite RS neurons with input from other neurons in the reticular formation and facilitate synaptic actions of these neurons. The diagram shows convergence on an RS neuron with an ipsilateral axon but would be equally valid for RS neurons with crossed descending projections and input from PT fibers of both sides.
A

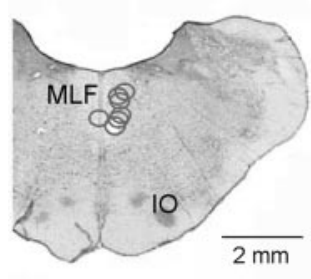

B

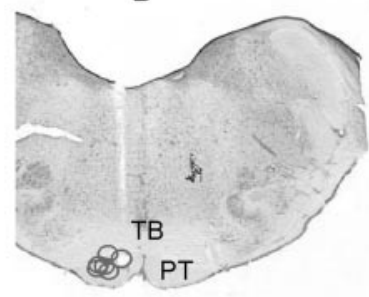

C

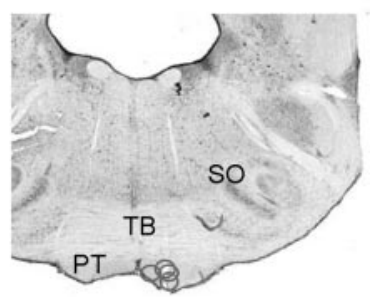

Figure 2. Reconstructions of the locations of the stimulating electrodes. $A-C$ show locations of electrode tips as defined by the electrolytic lesions made at the end of the experiments in the MLF and in the left and right PT, respectively. These are superimposed on representative sections of the brainstem cut in the plane of the electrode insertions. All MLF electrode placements were within the borders of the MLF, and all PT placements were within the pyramids or at the border with the TB. SO, Superior olive.

Santé, Antony, France). During recordings, neuromuscular transmission was blocked by pancuronium bromide $(\sim 0.2 \mathrm{mg} / \mathrm{kg} / \mathrm{hr}$, i.v.; Pavulon, Organon, Askim, Sweden), and the animals were artificially ventilated. Additional doses of $\alpha$-chloralose were given when increases in blood pressure or heart rate, which were continuously monitored, were evoked by noxious stimulation or if the pupils dilated. Mean blood pressure was kept at 100-130 $\mathrm{mmHg}$ and end-tidal concentration of $\mathrm{CO}_{2}$ at $\sim 4 \%$ by adjusting the parameters of artificial ventilation and the rate of a continuous infusion of a bicarbonate buffer solution with $5 \%$ glucose (1-2 ml/hr/kg). The core body temperature was kept at $\sim 37.5^{\circ} \mathrm{C}$ by servo-controlled infrared lamps. The experiments were terminated by a lethal dose of pentobarbital resulting in cardiac arrest.

A laminectomy exposed the fourth to seventh lumbar (L4-L7), low thoracic (Th11Th13), and in some experiments also second to fourth cervical (C2-C4) segments of the spinal cord. The spinal cord was hemisected on the left side in the Th12 segment (after removing the dorsal columns) at the beginning of the experiment. In some experiments, the dorsal parts of the lateral funiculi at the border between the C2 and C3 segments were sectioned bilaterally at a later stage to remove all corticospinal input to the spinal cord beyond this level (see Results). A number of peripheral hindlimb nerves were transected and mounted on stimulating electrodes. Subcutaneous cuff electrodes were used for nerves accessed in the iliac fossa [ipsilateral quadriceps (Q) and sartorius (Sart) nerves; the remaining ipsilateral nerves, the posterior biceps and semitendinosus (PBST) and anterior biceps and semimembranosus (ABSM); and gastrocnemius-soleus (GS)] were mounted on pairs of silver hook electrodes in a paraffin oil pool (at $36-37^{\circ} \mathrm{C}$ ) created by skin flaps.

The caudal part of the cerebellum was exposed by a craniotomy, and tungsten electrodes (impedance, $40-150 \mathrm{k} \Omega$ ) were placed in the right medial longitudinal fascicle (MLF) and in

Gossard et al., 1996), but whether these interneurons are activated by corticofugal fibers has not been investigated. Our second question was whether corticofugal fibers excite reticulospinal neurons, which affect motoneurons on the side of origin of the corticofugal fibers via ipsilaterally projecting premotor interneurons. Pathways that might permit this are as indicated in Figure $1 B$.

The results show that there is a strong facilitation of disynaptic actions of reticulospinal fibers on motoneurons, which permits the motor cortex to influence ipsilateral motoneurons. The effects mediated via commissural neurons are stronger than those mediated via ipsilateral premotor interneurons.

\section{Materials and Methods}

Preparation. The experiments were performed on seven deeply anesthetized cats weighing 2.3-3.5 kg. All experimental procedures were approved by Göteborg University Ethics Committee and followed National Institutes of Health and European Union guidelines for animal care. Anesthesia was induced with sodium pentobarbital ( $40-44 \mathrm{mg} / \mathrm{kg}$, i.p.) and maintained with intermittent doses of $\alpha$-chloralose (doses of 5 $\mathrm{mg} / \mathrm{kg}$ administered every $1-2 \mathrm{hr}$, up to $55 \mathrm{mg} / \mathrm{kg}$, i.v.; Rhône-Poulenc the left and/or right pyramidal tract (PT) in the low medulla (Fig. 2). The electrodes were inserted at an angle of $30^{\circ}$ (with the tip directed rostrally). The initial targets were at Horsley-Clarke coordinates P9, R0.6, and H-5 for MLF and P7, L1, H-10 and P5, R1, H-10 for the left and right PT, respectively. However, the final positions of the electrodes were adjusted on the basis of records of descending volleys evoked by single stimuli from the surface of the lateral funiculus at the Th11-Th13 and/or the C4-C5 segments (Fig. 3). The electrodes were left at sites from which distinct descending volleys were evoked at stimulus intensities of $\leq 20$ $\mu \mathrm{A}$. At the end of the experiments, these sites were marked with lesions $(0.3 \mathrm{~mA}$ constant current for $10 \mathrm{sec})$. Their location was subsequently verified on $100 \mu \mathrm{m}$ thick frontal sections of the brainstem, cut in the plane of insertion of the electrodes using a freezing microtome, and counterstained with cresyl violet. As shown in Figure 2, the MLF electrodes were placed at the level corresponding to the rostral border of the inferior olive (IO) (Fig. 2A) and the PT electrodes at the level of the caudal and rostral borders of the trapezoid body (TB) (Fig. $2 B, C$ ). Only data obtained in experiments in which the electrodes were appropriately placed will be reported.

Stimulation and recording. Peripheral nerves were stimulated with constant voltage stimuli [ $0.1 \mathrm{msec}$ duration; intensity expressed in multiples of threshold ( $\mathrm{T}$ ) for the most sensitive fibers in the nerve]. For 

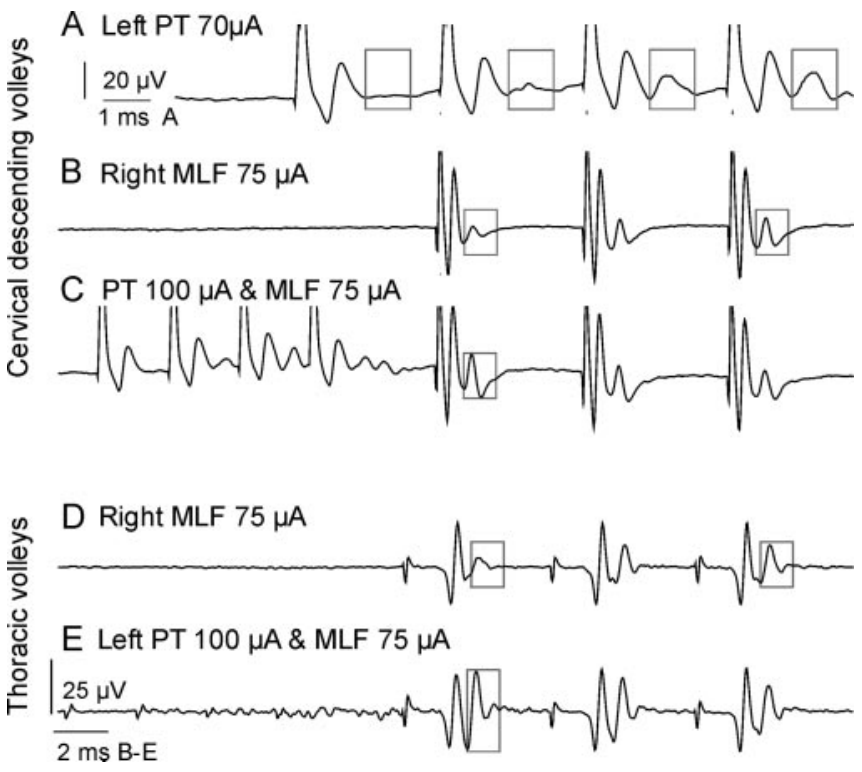

Figure 3. Descending volleys evoked by interacting stimulation of the right MLF and the left PT. A-C, Descending volleys recorded from the cord dorsum in the 44 segment (averages of 10 records). A shows the later components of volleys evoked by a train of PT stimuli (in the boxes) and the increase in the amplitudes of these components after each successive stimulus. $B$ shows a similar increase in amplitude of the later components of descending volleys evoked by a train of MLF stimuli (in box). C illustrates a large increase in the amplitude of the late component of the volley evoked by the first MLF stimulus (in box), when the MLF stimuli were preceded by a train of PT stimuli. $D$ and $E$ show a similar facilitation of late components of descending MLF volleys recorded at thoracic level. In this and the following figures, the largest stimulus artifacts have been truncated.

activation of fibers of the reticulospinal and corticospinal tract fibers, constant current cathodal stimuli (0.2 msec; $25-150 \mu \mathrm{A}$ in MLF, $100-$ $150 \mu \mathrm{A}$ for PT) were applied. Near maximal stimuli applied in MLF were expected to activate a large proportion of ponto and medullary reticulospinal tract fibers (Jankowska et al., 2003). These stimuli would also activate vestibulospinal tract fibers arising from the medial vestibular nucleus (which do not project caudally as far as the lumbar segments) but would not activate fibers from the lateral vestibular (Deiter's) nucleus (Nyberg-Hansen and Mascitti, 1964), so the effects in the lumbar segments can be attributed to reticulospinal fibers.

Descending volleys were recorded from the cord dorsum (at the lateral border of the dorsal columns at C3-C4 for PT volleys) or from the surface of the right lateral funiculus (at Th11-Th12 for MLF volleys) via intact dura mater, in both cases monopolarly.

Glass micropipettes filled with $2 \mathrm{~m}$ solution of potassium citrate were used for intracellular records from motoneurons. The motoneurons were identified by antidromic activation after stimulation of a muscle nerve, the ventral roots left intact. For the analysis of excitatory and inhibitory actions evoked from MLF, we preferentially sought GS and Sart motoneurons because disynaptic EPSPs and IPSPs were found previously to be mostly distinct and most frequently encountered in these motoneurons (Jankowska et al., 2003).

Analysis. Both the original data and averages of 10-20 single records were stored on-line. Any changes in the recorded potentials were estimated by comparing either the peak amplitudes or the areas of these potentials within selected time windows and also after having subtracted one from another (using a software sampling and analysis system designed by E. Eide, T. Holmström, and N. Pihlgren, Göteborg University, Göteborg, Sweden). Differences between samples of neurons were assessed for statistical significance using Student's $t$ test.

\section{Results}

To verify the existence of pathways between corticospinal tract neurons and ipsilateral motoneurons indicated in Figure $1 \mathrm{~A}$ and
$B$, all descending tract fibers were transected on one side of the spinal cord, and two experimental approaches were used. The first was to seek evidence for facilitation of the activation of reticulospinal tract fibers by conditioning stimulation of PT fibers. If this facilitation was not seen, it would be unlikely that the reticulospinal neurons projecting to the lumbosacral enlargement on the side opposite to the hemisection are excited by corticospinal neurons. However, depression or enhancement of the reticulospinal volleys would indicate interaction. A depression of the volleys might occur if the axons of RS neurons were discharged after activation by stimulation of PT fibers and made refractory. In contrast, if RS neurons were excited but not discharged by the stimulation of PT fibers, then they should be more excitable to activation through collaterals of MLF fibers, which could be reflected in an enhancement by spatial facilitation of the later components of the descending volleys. Observations related to these problems are presented below.

A second experimental approach was to analyze the effects of conditioning stimulation of PT fibers on disynaptic EPSPs and IPSPs evoked in hindlimb motoneurons by stimulation of axons of reticulospinal tract fibers in the MLF (Jankowska et al., 2003). These effects are described below.

\section{Evidence for PT actions evoked via RS neurons based on records of descending volleys}

Descending volleys evoked by stimulation of the medullary pyramid are usually difficult to see at thoracic and lumbar levels because of dispersion of the action potentials in fibers of different conduction velocities but are more readily recorded in the upper cervical segments (Illert et al., 1977; Lundberg, 1979). When recording cervical volleys evoked by a train of PT stimuli at 300$400 \mathrm{~Hz}$, in addition to the direct volleys that appeared $\sim 0.8 \mathrm{msec}$ after the stimuli, longer latency temporally facilitated components were seen (Fig. $3 A$, boxes). Because the differences in the onset latencies of the early and late components were $\sim 1 \mathrm{msec}$, the later components may be attributed to activation of additional neurons located somewhere between the PT and the recording site in the C3 segment. When submaximal PT stimuli were used, these longer latency volleys started to appear after the second or third stimulus and increased in amplitude after each successive stimulus (Fig. $3 A$ ), as would be expected for temporarily facilitated synaptic actions. Similar effects were seen in all four experiments in which cervical descending volleys were recorded. Our hypothesis is that the additional neurons mediating the longer latency volleys were RS neurons with axons in MLF, and we sought evidence for this by interacting the volleys evoked by PT stimuli with those evoked by MLF stimulation.

Stimulation of the MLF evokes volleys with early and late components (Jankowska et al., 2003). The early volleys are evoked by direct activation of descending RS axons and have constant amplitudes after each stimulus of the train. The later components of MLF volleys are considered to reflect transsynaptic activation of RS neurons via other RS neurons (Jankowska et al., 2003), as indicated in Figure $1 C$; consistent with this, their amplitudes increase with successive stimuli (Fig. 3 B). If stimulation of PT fibers evokes EPSPs in the same RS neurons, then the later components should be enhanced by spatial facilitation when both PT and MLF are stimulated together.

Figure $3 C$ shows that this facilitation was readily seen; the late component evoked by the first MLF stimulus was considerably larger when the MLF stimulus was preceded by a train of conditioning PT stimuli. The facilitation was clear in recordings made 
A MLF $150 \mu \mathrm{A}$

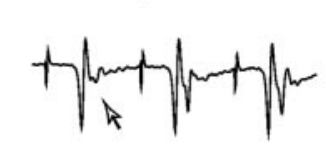

B Left PT $150 \mu$ A \& MLF before DLF lesions

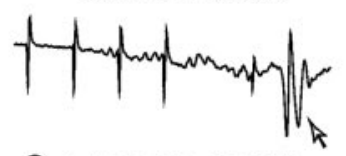

C Left PT $150 \mu$ A \& MLF
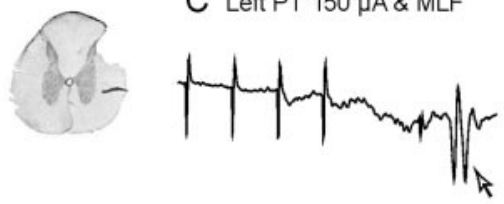

D Right PT $150 \mu \mathrm{A} \& \mathrm{MLF}$
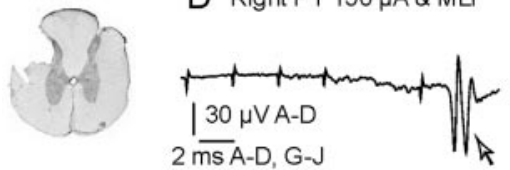

$2 \overline{\mathrm{ms} A}-\mathrm{D}, \mathrm{G}-\mathrm{J}$
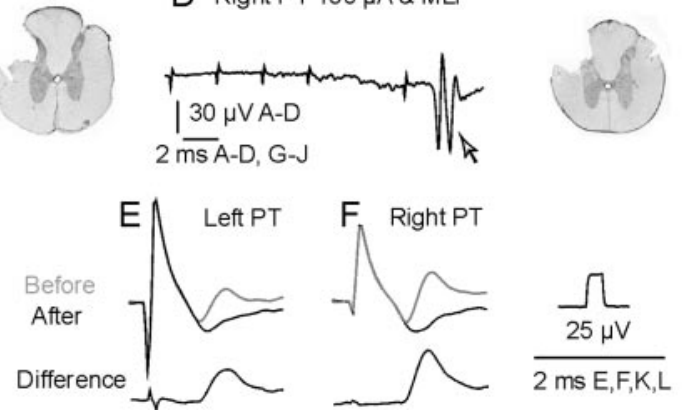

G
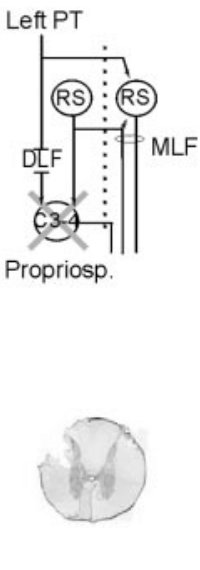

I Left PT $150 \mu$ A \& MLF $90 \mu \mathrm{A}$

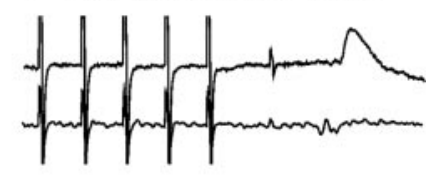

J Right PT $150 \mu A \&$ MLF $90 \mu \mathrm{A}$

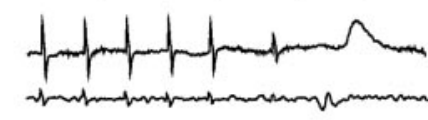

K Left PT

Figure 4. Facilitation of the late components of MLF descending volleys and of disynaptic EPSPs in motoneurons by stimulation of both left and right PT after elimination of corticospinal tract fibers at C2-C3.A, Descending volleys after stimulation of the right MLF with a train of three stimuli, recorded at the Th12 level (averages of 10 records). The arrow indicates the abortive late component evoked by the first stimulus. $B$, Effects of conditioning stimuli in the left pyramids on the volleys evoked by a single MLF stimulus (equivalent to the first stimulus shown in $A$ ) showing a large late component (arrow). ( and $D$ are the same as $B$ but after successively made lesions of the right and left DLF in the $(2$ segment, transecting connections between the left and right PT fibers and (3-C 4 propriospinal neurons as indicated in the diagram. The extent of the lesions is shown in the two sections ( $\sim 4 \mathrm{~mm}$ apart rostrocaudally) to the left. The disappearance of the PT volleys is illustrated in $E$ and $F$ where gray and black traces are records obtained before and after the lesions together with the differences between them. $E-H$, Intracellular records from a GS motoneuron after lesions of the dorsolateral funiculus in the ( 2 segment (shown to the left of / and J, with the corresponding control records in $K$ and $L$ ) in another experiment.

at both cervical and thoracic levels. Facilitation was also evoked from the right PT in all four experiments in which it was tested. The effects evoked from the right PT were either as strong (Fig. $4 D$ ) or slightly weaker than those from the left PT.

Late components of the descending volleys recorded at a thoracic level following PT and MLF stimuli might be relayed by brainstem reticulospinal neurons (Fig. 1C) but might also reflect the activation of spinal neurons, for example long propriospinal tract neurons with cell bodies in the $\mathrm{C} 3$ and $\mathrm{C} 4$ segments that are coexcited by corticospinal and reticulospinal tract fibers (Illert et al., 1981). To estimate the relative contributions of spinal and brainstem neurons, the facilitation of the later components of the descending volleys was compared before and after lesions of PT fibers in the upper cervical segments. Because PT fibers run in the most dorsal part of the lateral funiculus at the $\mathrm{C} 2$ level, they could be transected while leaving intact the reticulospinal tract fibers in the more ventral part of the lateral funiculus and in the ventral funiculus. These lesions were made following the procedure of Lundberg and collaborators (Illert et al., 1975; Lundberg, 1979), enlarged until the direct PT volleys recorded a few millimeters caudal to the lesions were abolished. The lesions were made bilaterally in two experiments. The extent of these lesions in

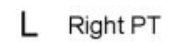

two experiments is shown in Figure $4 C$, $D, G$, and $H$, and the resulting disappearance of PT volleys in the lowest panels $(E, F, K, L)$. Because the PT descending volleys recorded at the $\mathrm{C} 3-\mathrm{C} 4$ segments are evoked at latencies of $<1$ msec and are superimposed on fairly large stimulus artifacts, they can be more conveniently estimated from the difference records obtained before and after dorsolateral funiculus (DLF) lesions.

The records in Figure $4 A-D$ show that temporal facilitation of late components of MLF volleys occurred and that spatial facilitation was evoked when a single MLF stimulus was delivered after a train of conditioning stimuli applied within either the left or the right pyramidal tracts. The arrows indicate the late components evoked by single MLF stimuli recorded from the thoracic segments. As in Figure 3, facilitation from the ipsilateral (left) PT was more potent than facilitation by two or even three MLF stimuli. Facilitation from the contralateral (right) PT was also substantial. The records in Figure $4 C$ and $D$ were obtained after lesions of the dorsal parts of the lateral funiculi.

In one of the experiments, the effects of the conditioning PT stimuli were tested not only on the descending volleys but also on EPSPs evoked in motoneurons. In all 10 motoneurons recorded intracellularly after DLF lesions, PT stimuli (both ipsilateral and contralateral) continued to facilitate disynaptic EPSPs evoked from MLF (see below), as illustrated in Figure $4 I$ and $J$. In this case, the effects have to be relayed via commissural neurons, and to bring these to threshold, a train of two or three MLF stimuli were required. Accordingly, the train of stimuli to the PT was delivered before the final stimulus of a train of stimuli to the MLF, rather than to a single MLF stimulus. Thus, both of these control experiments indicate that facilitatory effects of PT stimuli are associated with actions on RS rather than on propriospinal neurons in the C3-C4 segments or, more generally, on any spinal neurons located caudal to these segments.

In contrast to the late components of the MLF descending volleys, the early components of the volleys were either depressed or unaffected by PT stimuli. The depression can be explained by refractoriness: if collaterals of PT neurons excited reticulospinal neurons with axons in MLF sufficiently strongly to discharge them, they should be refractory to direct activation by MLF stimuli at short conditioning testing intervals. The results of three experiments in which amplitudes of the early and later components of the same MLF volleys were plotted as a function of the intervals between the last PT stimulus of a train and an MLF stimulus are shown in Figure $5 A-C$ and exemplified in Figure $5 F$. The black symbols in $A-C$ show that the early (i.e., direct) components of the descending MLF volleys recorded at the thoracic and lumbar levels were decreased by approximately one-half at optimal conditioning-testing intervals but did not disappear. 
The maximal decrease of the thoracic volleys occurred at conditioning-testing intervals of $2-2.5 \mathrm{msec}$. We estimate that $\sim 1.5-1.8$ msec might be needed for RS neurons to be activated by nerve impulses in collaterals of corticospinal tract fibers after PT stimuli (allowing $\sim 0.5 \mathrm{msec}$ for conduction time along these collaterals, $0.3 \mathrm{msec}$ for synaptic delay, and up to 1 msec for the rise time of the asynchronously evoked EPSPs before action potentials are generated) and that the conduction time between the RS neuron somata and the site of application of the stimuli in MLF might be another 0.2 msec. The period during which the early MLF volleys were decreased is thus appropriate to coincide with the refractory periods of the axons of RS neurons. Several factors may explain why the volleys were only partly decreased but not abolished, for instance if the activation of reticulospinal neurons by PT stimulation was asynchronous, or only a fraction of RS neurons was activated (and therefore refractory), or if corticospinal neurons might provide input to only a proportion of the RS neurons that have axons in the MLF. The proportionally smaller decreases of the earliest components of MLF volleys recorded at a cervical level (compare simultaneously recorded thoracic and cervical volleys in Fig. $5 G, H, E, F)$ suggest that a smaller proportion of the RS neurons that terminate within the cervical or upper thoracic segments is discharged by corticospinal neurons.

If the facilitation of the late components of MLF volleys is attributable to PT actions on RS neurons, then they ought to be reduced during the expected refractory period after activation of RS neurons like the early components. This was indeed the case, as indicated by the gray data points in Figure $5 A, B$, and $D$. The onset of the depression of the late components was sharper, if not earlier, than the onset of the depression of the early components. The degree of depression was likewise similar, to approximately one-half of control amplitude.

Although these results are generally in keeping with activation of RS neurons by PT fibers, they are less clear cut than the results of similar tests on the activation of vestibulospinal neurons by stimuli applied in the cerebellum (Matsuyama and Jankowska, 2004) where the experimental situation was much simpler. The main complicating factor in the present study was that activation of RS neurons by PT fibers was relatively weak. Accordingly, a train of PT stimuli was needed, and the effects of the final PT stimulus of a train were often superimposed on weak depressive (on direct volleys) and stronger facilitatory (on later volleys) effects of the penultimate stimulus. For the early components of the volleys, this resulted in two periods of depression related to the refractory periods after the third and fourth stimuli of the PT train, as indicated in Figure $5 A-C$ by open and filled symbols, respectively.

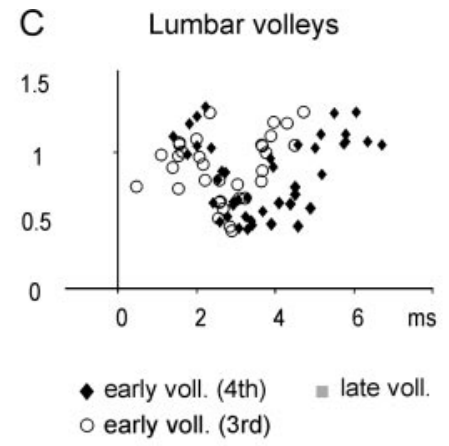

D Cervical volleys (single MLF)

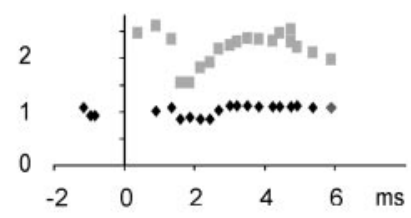

Figure 5. Effects of a stimulation of the left PT on descending volleys evoked by subsequent stimulation of the MLF. $A$ and $B$ plot Hz) from two experiments. The $x$-axis labels refer to the time between the final PT stimulus and the MLF stimulus. The (t) attributable to the effects of the fourth PT stimulus. The open symbols show changes in the early components that are attributable to the third stimulus of the train to the PT. The gray symbols represent the late components. $C$ and $D$ show similar data for the largest changes in volleys recorded at the $\mathrm{L} 6$ and C4 segmental levels (from the same experiment as the plots in $A$ and $C$, respectively). Note that in C, the time intervals for facilitation and occlusion evoked by the third and fourth stimulus are superimfourth PT stimulus (filled symbols). E, F, Examples of test and conditioned volleys recorded at thoracic level at a conditioning testing interval, indicated by the double arrow, at which the early components of the volleys following the first MLF stimulus (boxed) were most effectively depressed. G, H, Test and conditioned volleys simultaneously recorded at cervical level. Voll., Volley.

Evidence for ipsilateral PT actions evoked via RS neurons and commissural lamina VIII interneurons based on intracellular records from motoneurons

Reticulospinal tract neurons evoke disynaptic EPSPs and IPSPs via commissural neurons in a particularly high proportion of contralateral GS and Sart motoneurons, respectively (Jankowska et al., 2003). Intracellular recordings were therefore sought principally from GS and Sart motoneurons but also included other motoneuron types to test whether any PSPs evoked in them from MLF could be facilitated by PT stimuli. The experimental arrangement was as outlined in Figure $1 A$, and intracellular recordings were made from motoneurons ipsilateral to the spinal hemisection. Disynaptic PSPs were evoked by two or three MLF stimuli and, after adjusting the intensity of these stimuli such that at least the first one was sub- or near-threshold, were interacted with PT stimuli. Trains of PT stimuli were used for conditioning because, as shown above, two to four stimuli were needed to facilitate synaptic activation of reticulospinal neurons. The 
A Right MLF $25 \mu \mathrm{A}$

\section{Left PT $100 \mu \mathrm{A}$} right MLF $25 \mu \mathrm{A}$
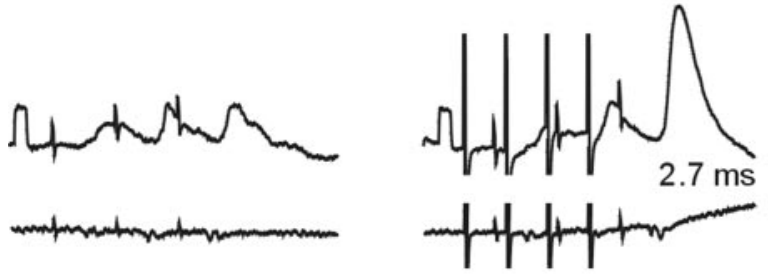

B Right MLF $50 \mu \mathrm{A}$

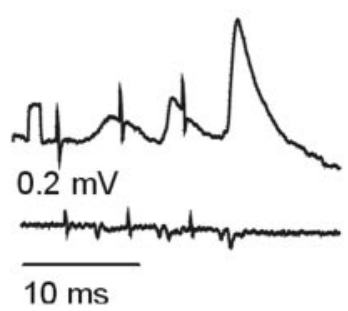

D

Right PT $100 \mu \mathrm{A}$ right MLF $25 \mu \mathrm{A}$

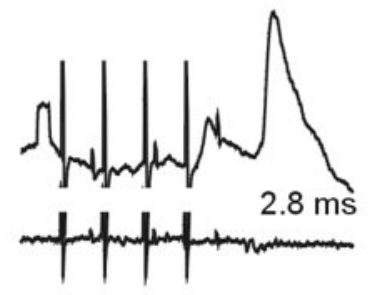

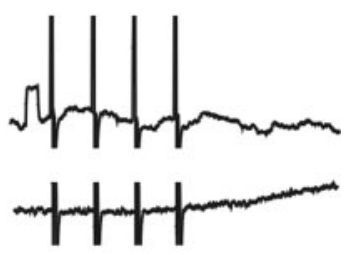

E Left PT $100 \mu \mathrm{A}$

F Right PT $100 \mu \mathrm{A}$

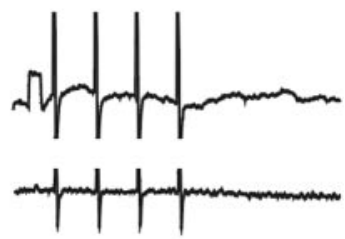

Figure 6. Facilitation of disynaptic EPSPs evoked from the MLF by conditioning stimulation of the pyramids. Top records in each panel are intracellular potentials from a GS motoneuron on the left side of the spinal cord; the bottom records are from cord dorsum. Voltage calibration ( $0.2 \mathrm{mV}$ for the calibration pulse at the beginning of the records) in $B$ is for the intracellular records. The largest stimulus artifacts have been truncated. $A$, Test EPSPs evoked by stimulation of the right MLF with a train of three stimuli that were too weak to produce a temporal facilitation. $B$, Temporal facilitation of EPSPs evoked by stronger MLF stimuli. C, D, Facilitation of effects of weak MLF stimuli when the left $(C$ or right $(D)$ PT were also stimulated with a train that terminated $\sim 5$ msec before the final MLF stimulus. $E$, $F$, Negligible actions of stimuli to the left and right PT when stimulated alone.

EPSPs and IPSPs in motoneurons were the result of spatial and temporal facilitation at the level of both reticulospinal and spinal commissural interneurons.

Potent facilitation of EPSPs of MLF origin could be evoked from both the left and right pyramids, as illustrated in Figure $6 C$ and $D$. The effects of PT stimuli were in this case tested on EPSPs evoked at such a low stimulus intensity $(25 \mu \mathrm{A})$ (Fig. $6 \mathrm{~A})$ that they showed marginal temporal facilitation. Distinct temporal facilitation was evoked when stronger MLF stimuli (50 $\mu \mathrm{A}$ ) (Fig. $6 B)$ were used. Conditioning stimulation of the left or the right PT coupled with MLF stimulation evoked EPSPs that were greatly facilitated (Fig. 6C,D), exceeding the amplitude of those evoked by a train to the MLF at $50 \mu \mathrm{A}$. Because the PT stimuli at this intensity did not evoke any PSPs when delivered alone (Fig. $6 E, F)$, facilitation must have occurred at a premotoneuronal level.

In order for facilitation to occur, several conditions had to be fulfilled. Facilitation was found when the test EPSPs were near threshold and, as illustrated in Figure $7 E$ and $F$, was much more potent when trains of four (rather than three) PT stimuli were used. The frequency of the train of PT stimuli was critical, because facilitation was weak with trains at $200 \mathrm{~Hz}$ but was more effective with trains at 300 or $400 \mathrm{~Hz}$. The difference between effects of 200 and $300 \mathrm{~Hz}$ stimuli is illustrated in Figure 7D and $E$. Finally, the time intervals between the final stimuli of the trains to the PT and MLF had to be appropriate.

Two example plots of the time course of facilitation of the EPSPs are shown in Figure 8. The most consistent feature of these plots was that the peak of the facilitation occurred at an interval of $\sim 5$ msec between the final stimulus of the train to the PT and the MLF stimulus (as in Fig. $8 A$ ). The peak effect thus occurred $\sim 1$ msec later than the peak of facilitation of the MLF volleys that are illustrated in Figure 5. The precise onset of the facilitation was difficult to define, even in the plots with the sharpest rising slopes (as in Fig. $8 \mathrm{~A}$ ). It began at conditioningtesting intervals of $\sim 2-3 \mathrm{msec}$ (i.e., closely following the time of onset of the facilitation of MLF volleys). Failure of facilitation, which would correspond to the refractory period (at intervals $1.5-2.5 \mathrm{msec}$ ) in the plots of the MLF volleys in Figure 5, was not seen. However, some facilitation could have been induced by the third as well as the fourth PT stimulus, which might mask any effects of the refractory period after the fourth stimulus. The time courses of facilitation of EPSPs recorded in nine other motoneurons and of IPSPs recorded in another motoneuron were similar.

Using the optimal stimulus parameters and intervals (4-7 msec) between conditioning stimuli applied within the left (ipsilateral) pyramid and test stimuli to the right (contralateral) MLF, facilitation of disynaptic EPSPs could be demonstrated in all motoneurons tested. These included 15 GS, 5 Q, 1 PBST, 1 Sart, and 3 unidentified motoneurons. The facilitated EPSPs evoked by the third MLF stimulus of a train, measured from the area within a time window of 1.5-2.0 msec from the onset, had amplitudes up to 8-10 times larger than the areas of test EPSPs. The mean increase was $3.04 \pm 0.38$ times (mean \pm SEM; $n=25$ ) (Fig. 9A). Conditioning stimulation of the right (contralateral) pyramid produced a similar degree of facilitation (increasing EPSPs evoked in the same 25 motoneurons $2.75 \pm 0.38$ times). Disynaptic EPSPs were also facilitated in 10 additional motoneurons recorded after lesions of the descending corticospinal tract fibers between the C2 and C3 segments, indicating that the facilitation occurred supraspinally. Records from one of these neurons are illustrated in Figure $4 E-H$.

The records in Figure 10 show that IPSPs evoked in motoneurons by the MLF stimuli were also facilitated by ipsilateral PT stimuli. In this case, a train of three near-maximal stimuli to the MLF evoked small disynaptic IPSPs (Fig. 10 A), but distinct larger IPSPs appeared on joint stimulation of the MLF and a train of stimuli to the left or right PT at $150 \mu \mathrm{A}$ (Fig. $10 \mathrm{~B}, \mathrm{D}$ ), whereas PT stimuli by themselves were ineffective (Fig. 10C,E). Facilitation was found in all of the 16 motoneurons tested, including 2 GS, 3 $\mathrm{Q}$, and 11 Sart motoneurons. On average, the facilitated IPSPs were $4.83 \pm 0.87$ times larger than the test IPSPs (Fig. 9A). The effects of stimulation of the left and right pyramids were compared in six of these motoneurons. There were no significant differences between the effects evoked from left and right side, although there was a tendency for the IPSPs that followed stimulation of the ipsilateral (in this case, left) PT to be somewhat larger (Fig. 9A).

\section{Evidence for ipsilateral PT actions involving RS neurons but not commissural lamina interneurons}

Reticulospinal tract neurons may act on various populations of spinal interneurons in addition to lamina VIII commissural interneurons, some of which are premotor interneurons that act on 


\section{A Right MLF $25 \mu \mathrm{A}$}

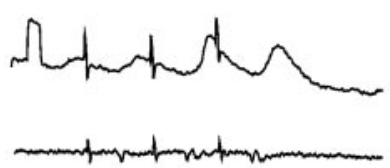

B Right MLF $50 \mu \mathrm{A}$
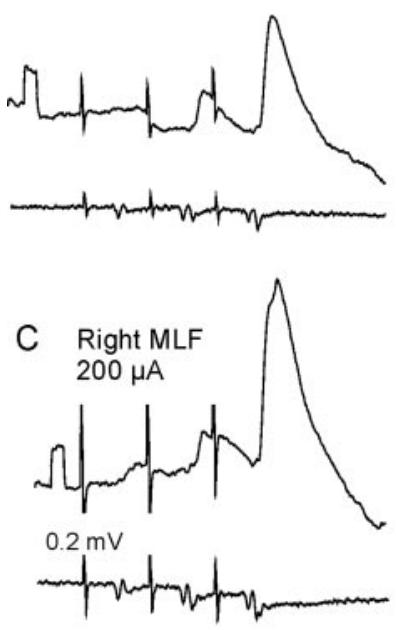

D Left PT $100 \mu \mathrm{A}$ right MLF $25 \mu \mathrm{A}$

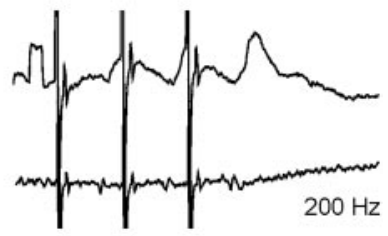

E Left PT $100 \mu \mathrm{A}$ right MLF $25 \mu \mathrm{A}$

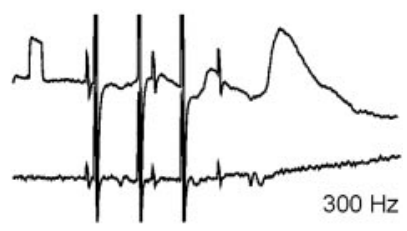

F Left PT $100 \mu \mathrm{A}$ right MLF $25 \mu \mathrm{A}$
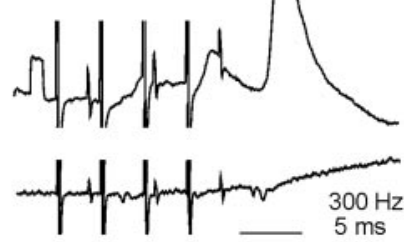

Figure 7. Parameters of PT stimulation needed for effective facilitation of synaptic actions evoked from MLF. The top records in each panel are from a GS motoneuron on the left side of the spinal cord. The bottom records are from the cord dorsum. $A-C$, Small EPSPs were evoked in this motoneuron by a train of three MLF stimuli at $25 \mu \mathrm{A}$, and considerable temporal facilitation was evident with stronger stimulation (50 and $200 \mu \mathrm{A}$ ). D-F, Spatial facilitation of the EPSPs evoked by trains of stimuli to the PT at different frequencies. Note that three PT stimuli at $200 \mathrm{~Hz}$ evoked only marginal facilitation of the EPSP evoked by the third MLF stimulus, and that the effectiveness of the PT stimuli was much greater when their frequency increased from 200 to $300 \mathrm{~Hz}(E)$, and in particular when a fourth stimulus was added $(F)$. Otherwise, the format is the same as in Figure 6.

motoneurons located on the same side of the spinal cord (Takakusaki et al., 1989; Davies and Edgley, 1994). Another possible route for ipsilateral actions evoked by corticospinal neurons thus might be via ipsilaterally projecting RS neurons and ipsilaterally projecting interneurons. To explore this possibility, the effects of stimulation of the right pyramid were tested on 26 motoneurons located on the right side of the spinal cord in preparations in which the spinal cord was hemisected on the left side (the same preparations in which the effects from the left pyramids described above were investigated, as indicated in the diagram of Fig. $1 B$ ). Under these conditions, facilitation of disynaptic EPSPs and IPSPs evoked by MLF stimulation was more difficult to estimate because the MLF stimuli sometimes evoked monosynaptic EPSPs in motoneurons (as in the records in Fig. 11A-C). However, in motoneurons in which they were distinct, disynaptic EPSPs $(n=$ $6)$ and IPSPs $(n=9)$ were increased $1.13 \pm 0.13$ and $2.10 \pm 0.45$ times, respectively, by conditioning stimulation of the right (ipsilateral) PT. The degree of facilitation of EPSPs and IPSPs mediated by ipsilateral premotor interneurons thus appeared to be somewhat weaker than the facilitation of PSPs mediated by commissural interneurons (Fig. 9, compare $A$ and $B$ ).

Although facilitation of disynaptic EPSPs by conditioning stimulation of the left and right PT was similar in motoneurons

on the right (Fig. $11 B, C$ ), facilitation of disynaptic IPSPs was much more potent from the left PT than from the right PT (Fig. $11 E, F)$. Thus, stimulation of corticospinal tract fibers from the left PT (which descended on the intact right side of the spinal cord) increased these IPSPs more than 10-fold (Figs. 9B, 11E), and a strong effect was seen in almost all motoneurons. In contrast, these IPSPs were facilitated to about the same extent as the disynaptic EPSPs by stimulation of the right PT. The asymmetry in these observations also provides evidence that the effects of stimuli that we used may be attributed to the fibers in the stimulated pyramid, even if stronger stimuli might have activated some fibers on the opposite side.

\section{Discussion}

\section{Pathways from the corticospinal tract to} ipsilateral motoneurons

The results provide new observations on indirect pathways via which corticospinal tract neurons may excite or inhibit ipsilateral hindlimb motoneurons and show that these pathways involve reticulospinal tract neurons with axons in MLF and lamina VIII excitatory and inhibitory commissural interneurons.

In these experiments, all direct actions of ipsilaterally descending fibers, including the small proportion of corticospinal fibers that descend ipsilaterally (Armand and Kuypers, 1980; Armand et al., 1985; Li and Martin, 2000; Lacroix et al., 2004), were eliminated by hemisection of the spinal cord. Some of the crossed corticospinal axons descending on the intact side might have contributed by decussating below the hemisection (Armand and Kuypers, 1980; Armand et al., 1985; Li and Martin, 2000; Lacroix et al., 2004), but they were not essential because additional bilateral corticospinal tract lesions of the DLF in upper cervical segments (Fig. 4) did not affect PSPs evoked in ipsilateral motoneurons. This leads to the conclusion that the main relay of the corticospinal actions we observed in ipsilateral motoneurons was in the brainstem and the descending pathway that mediated them was not the corticospinal tract. The persistence of ipsilateral PT actions after DLF lesions also eliminates an essential contribution from spinal neurons located rostral to these lesions, including the long propriospinal tract neurons in the C3-C5 segments, which are coexcited by both PT and RS neurons (Alstermark et al., 1987).

Given that the actions of ipsilateral corticospinal neurons are mediated by brainstem neurons, the two most likely relays are via reticulospinal or rubrospinal neurons. However, the upper cervical DLF lesions would also have transected rubrospinal fibers, arguing against a major contribution from them. Furthermore, rubrospinal fibers decussate on exiting the nucleus before descending, and there is no information on potential ipsilateral actions adding to strong contralateral actions (Hongo et al., 1969).

A major role can thus be attributed to reticulospinal neurons. Three lines of evidence support this conclusion. First, there is substantial morphological evidence for bilateral projections from corticospinal tract neurons to reticular formation neurons (Keizer and Kuypers, 1984, 1989; Ugolini and Kuypers, 1986; Matsuyama and Drew, 1997; Kably and Drew, 1998) and direct electrophysiological evidence for excitation of reticulospinal cells by PT fibers (He and Wu, 1985; Canedo and Lamas, 1993), which is supported by our observations that descending volleys evoked by stimulation of axons of RS neurons are substantially facilitated by PT stimulation. Second, stimulation of the ipsilateral pyramid produced effects that were very similar to the actions of reticulospinal neurons reported previously (Jankowska et al., 2003). 


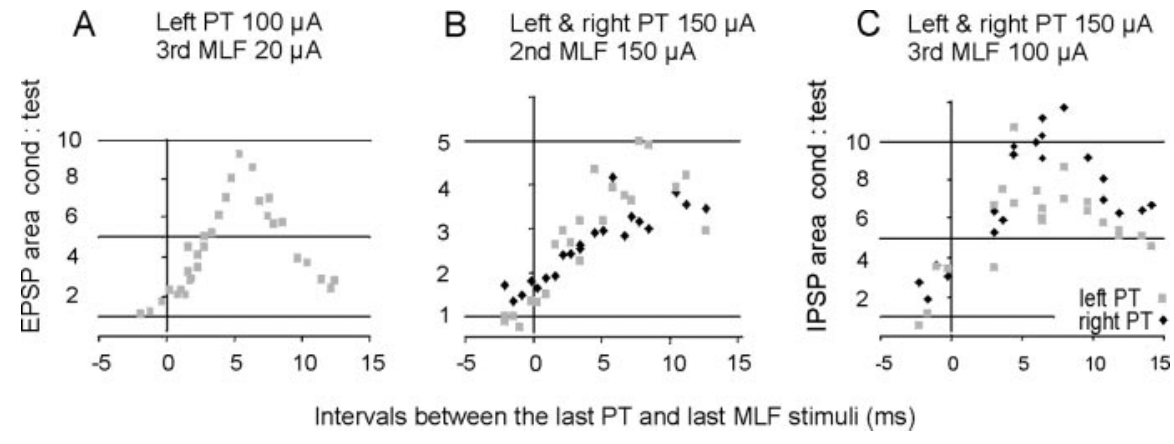

Figure 8. Time course of facilitation of disynaptic PSPs evoked from MLF by stimulation of pyramidal tract fibers. $A, B$, Time course of facilitation of EPSPs evoked in GS motoneurons on the left side of the spinal cord after stimulation of the left and right pyramids indicated by gray and black symbols, respectively. C, Similar data for IPSPs evoked in another GS motoneuron. Intervals were measured between the fourth PT stimulus and the third MLF stimulus. The area of the PSPs was measured within 2 msec time windows from their onset. Time 0 on the $x$-axis represents the timing of the effective MLF stimulus, relative to the last PT conditioning stimulus. cond, Conditioned.

B Right motoneurones

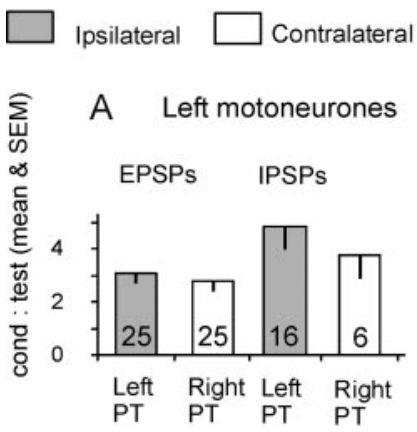

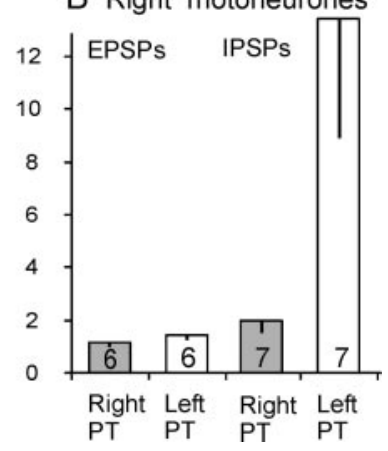

Figure 9. Comparison of the effectiveness of facilitation of PSPs evoked from the right MLF in motoneurons on the left $(A)$ and right $(B)$ side of the spinal cord by conditioning stimulation of the left and right PT. The bars represent the mean amount of facilitation [ratio of conditioned (cond) to test] with SE bars. $A$, Facilitation in motoneurons on the left (via commissural interneurons). $B$, Facilitation in motoneurons on the right (via ipsilaterally projecting neurons). Facilitatory actions of left and right pyramids (abscissa) were tested on the same motoneurons and using the same parameters of stimulation. The only statistically significant differences (Student's $t$ test; $p=0.04$ ) between the effects evoked from the left and right PT were those on IPSPs evoked in the right side motoneurons.

Third, the relay is in the brainstem where the reticulospinal neurons are located.

Nevertheless, we cannot exclude that pathways via propriospinal or rubrospinal neurons may contribute to the overall effects of PT stimulation in parallel with reticulospinal neurons in preparations with DLF intact. The similarity of the effects after DLF lesions suggests that, if present, these contributions are minor. However, experiments in which corticospinal effects would be tested after lesions of the reticular formation or reticulospinal tract fibers and leaving only the $\mathrm{C} 3-\mathrm{C} 5$ propriospinal neurons and rubrospinal neurons intact were not performed.

Because the left side of the spinal cord was transected above the lumbosacral enlargement, the effects of stimulation of the pyramids might be attributed to relays via lumbar commissural interneurons at the right side. An alternative route would be via the small number of reticulospinal tract axon collaterals that cross the midline at a lumbar level (Matsuyama et al., 1988, 1997), which might contact motoneurons, although this is rare (Jankowska et al., 2003), or activate premotor interneurons located on the same side as the motoneurons. However, because the number of reticulospinal neurons activated by pyramidal tract fibers would be even smaller than the number activated by stimuli applied in the MLF, the probability of activating ipsilaterally located premotor interneurons by axon collaterals crossing at segmental level would be considerably lower than in the case of more directly activated RS neurons (for other arguments, see Jankowska et al., 2003). Candidate commissural neurons that form synapses with contralateral motoneurons have been described in the midlumbar segments of the spinal cord (Bannatyne et al., 2003; Jankowska et al., 2003; Hammar et al., 2004).

The results of this study suggest that the minimal coupling between corticospinal tract neurons and ipsilateral motoneurons via commissural interneurons is trisynaptic, assuming that the RS neurons that activate lumbar commissural interneurons are monosynaptically activated by collaterals of PT fibers ( $\mathrm{He}$ and $\mathrm{Wu}, 1985$; Canedo and Lamas, 1993; Matsuyama and Drew, 1997). The latencies of the late, temporally facilitated components of PT volleys were in keeping with monosynaptic activation of descending tract neurons $(\sim 1 \mathrm{msec})$, and the onset of facilitation of the second components of MLF volleys and the disynaptic EPSPs and IPSPs of MLF origin was appropriate for direct actions of PT fibers on RS neurons.

The proposed basis for facilitation of activation of RS neurons is shown in Figure $1 C$. This depends on temporal facilitation of the actions of successive nerve impulses in PT fibers and of mutual spatial facilitation between these actions and synaptic actions evoked by the MLF stimuli via axon collaterals of reticulospinal or locally projecting reticuloreticular neurons. Although the specific connections remain unknown, Canedo and Lamas (1993) showed that many reticulospinal neurons were excited by corticofugal fibers at latencies longer than those of the monosynaptic EPSPs and were considered to be generated disynaptically. In addition, MLF stimulation generates monosynaptic EPSPs in a high proportion of reticular neurons (Ito and McCarley, 1987; McCarley et al., 1987). If this excitation was mediated by recurrent axon collaterals of RS neurons, then it would increase the number of recruited RS neurons by a kind of positive feedback. In the diagram in Figure $1 C$, the collaterals shown arise from reticulospinal neurons with cell bodies located either on the same side or opposite side; both combinations seem to be possible (Matsuyama et al., 1988, 1993). Another possibility might be that the PT collaterals act not on RS neurons but on other reticular formation neurons that provide input to the RS neurons. However, as shown in Figures $2 C$ and $E$ and $4 B, C$, and $D$, the later components of descending MLF volleys were facilitated to a greater extent by PT stimuli than by MLF stimuli, suggesting that input from PT fibers to RS neurons might be stronger than input via RS recurrent collaterals and more compatible with direct than with relayed actions of PT fibers.

\section{Functional considerations}

The demonstration of a trisynaptic pathway through which corticospinal neurons can influence ipsilateral motoneurons provides a new potential for the treatment of motor dysfunctions after unilateral injuries of the corticospinal tract (e.g., after stroke, tumors, or other injuries at supraspinal or spinal levels). If crossed corticospinal actions become deficient, recovery of func- 
tion might involve ipsilateral actions of intact corticospinal neurons, as indicated by both clinical observations (Weiller et al., 1992; Feydy et al., 2002; Strens et al., 2003) and behavioral experiments in animals (Kucera and Wiesendanger, 1985; DarianSmith et al., 1996; Whishaw et al., 1998; Liu and Rouiller, 1999; Pettersson et al., 2000). If these ipsilateral actions, which are normally much weaker than contralateral actions, can be enhanced at spinal and/or bulbar levels, then this represents a new opportunity to restore function. There is already evidence for spinal "detour circuits" that involve plastic changes in spinal interneuronal circuits and their input connections (Galea and DarianSmith, 1997; Bareyre et al., 2004). Identification of a route through reticulospinal tract neurons and commissural neurons that can mediate ipsilateral corticospinal actions provides another potential "detour" pathway as a target for intervention. Previous studies have revealed that activation of commissural interneurons is powerfully modulated by presynaptic inhibition (Edgley et al., 2003) and by monoamines (Hammar et al., 2004), both of which might provide a potential means for influencing their excitability (e.g., pharmacologically). Modulation by serotonin and noradrenaline represents a particularly interesting possibility, because both enhance the activation of commissural interneurons by reticulospinal fibers (Hammar et al., 2004). However, serotonin and noradrenaline also act on other spinal interneurons and motoneurons, and their effects at supraspinal level (e.g., on the reticulospinal neurons) would also have to be considered. Another possibility might be to use different rehabilitation procedures to raise and maintain activity in pathways involving commissural interneurons at a high level, facilitating their activation by descending commands. Again, our knowledge of how this could be achieved will depend on analysis of the functional connections of the commissural interneurons. Finally, although the pathways using ipsilaterally located interneurons were less effective than commissural pathways, they also represent a potential route for the recovery of function. An understanding of the neuronal networks that mediate connections between corticospinal neurons and ipsilateral motoneurons should be of clinical use in both rehabilitation and pharmacological procedures.

\section{References}

Alstermark B, Pinter M, Sasaki S (1983) Brainstem relay of disynaptic pyramidal EPSPs to neck motoneurons in the cat. Brain Res 259:147-150.

Alstermark B, Lundberg A, Pinter M, Sasaki S (1987) Subpopulations and functions of long C3-C5 propriospinal neurones. Brain Res 404:395-400.

Alstermark B, Pinter MJ, Sasaki S (1992) Descending pathways mediating disynaptic excitation of dorsal neck motoneurones in the cat: brain stem relay. Neurosci Res 15:42-57.
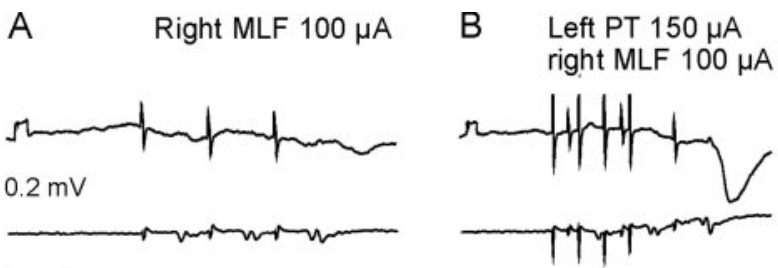

C Left PT $150 \mu \mathrm{A}$

Figure 10. Facilitation of disynaptic IPSPs evoked from MLF by conditioning stimulation of pyramids. Top records in each panel are from a GS motoneuron on the left side. Bottom records are from cord dorsum. Otherwise, the format is the same as in Figure 6.
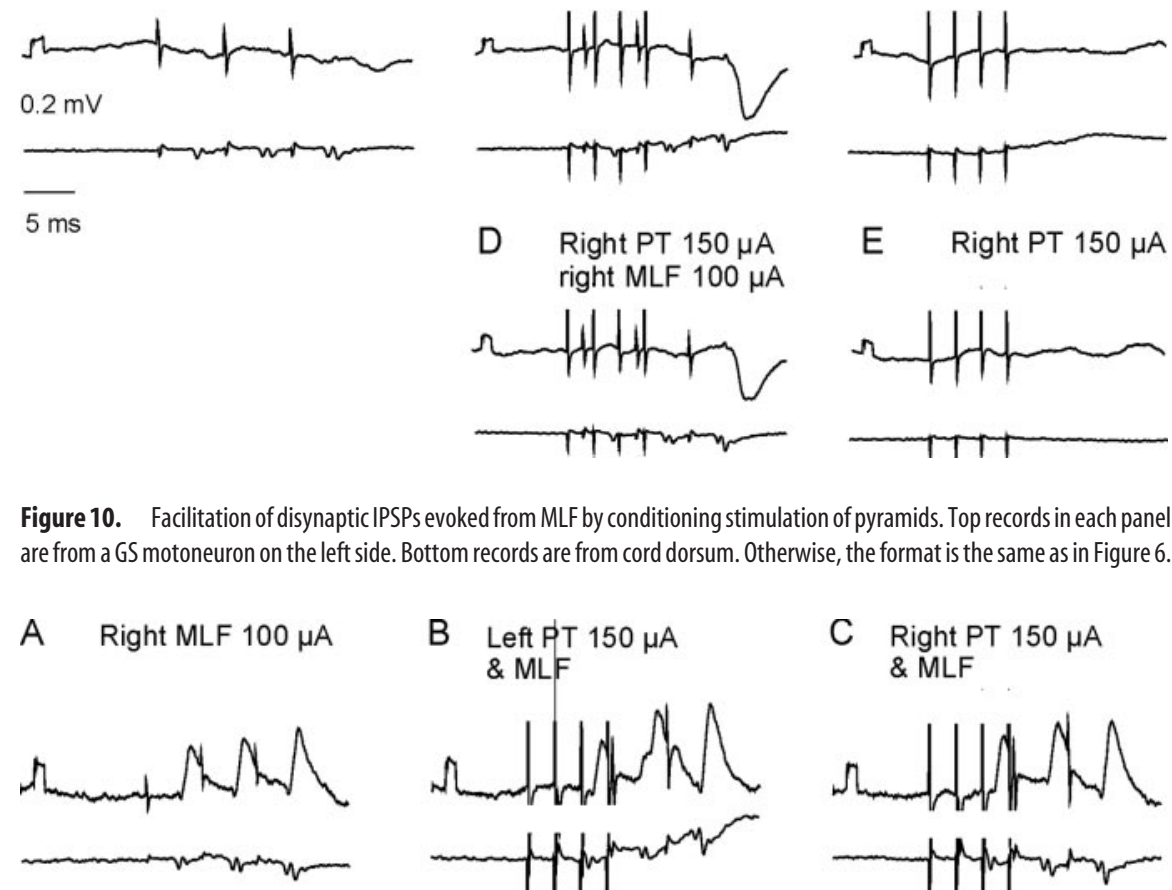

E Right PT $150 \mu \mathrm{A}$

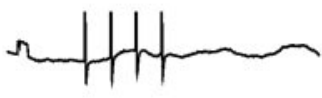

TTTा
D Right MLF $150 \mu \mathrm{A}$

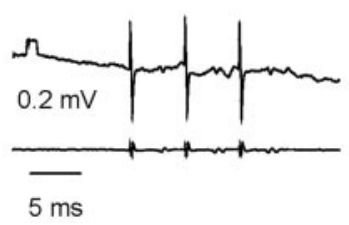

Figure 11. Facilitation of disynaptic EPSPs and IPSPs in motoneurons on the right side of the spinal cord. Top records in each panel are intracellular records from a motoneuron on the right side of the cord. $A-C$, From a GS motoneuron; $D-F$, from a 0 motoneuron. Bottom records are from the cord dorsum. $A$ and $D$ show the responses of the motoneurons to a train of three stimuli to the MLF. $B, C, E$, and $F$ show the effects of a train of PT stimuli delivered to end 5-10 msec before the final MLF stimulus. The lowermost records in $B, C, E$, and $F$ are as the top records but showing effects of $P T$ stimuli alone. Otherwise, the format is the same as in Figure 6 (the figure illustrates effects summarized in Figure $9 B$ ).
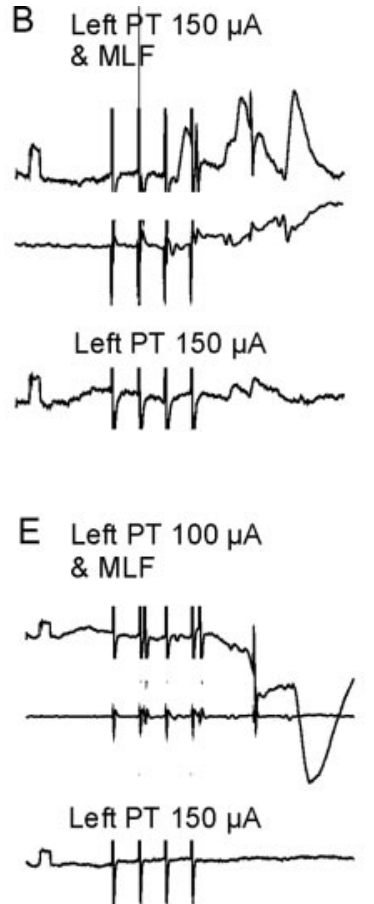

C Right PT $150 \mu \mathrm{A}$ \& MLF

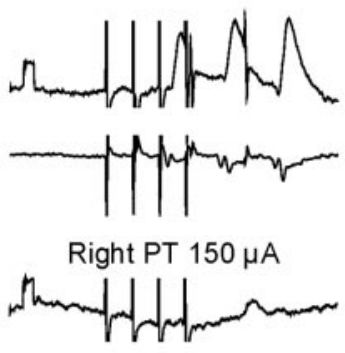

F Right PT $150 \mu \mathrm{A}$ \& MLF
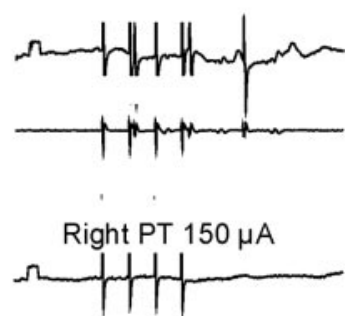

Armand J, Kuypers HG (1980) Cells of origin of crossed and uncrossed corticospinal fibers in the cat: a quantitative horseradish peroxidase study. Exp Brain Res 40:23-34.

Armand J, Holstege G, Kuypers HG (1985) Differential corticospinal projections in the cat. An autoradiographic tracing study. Brain Res 343:351-355.

Bannatyne BA, Edgley SA, Hammar I, Jankowska E, Maxwell DJ (2003) Networks of inhibitory and excitatory commissural interneurons mediating crossed reticulospinal actions identified by immunocytochemistry. Eur J Neurosci 18:2273-2284.

Bareyre FM, Kerschensteiner M, Raineteau O, Mettenleiter TC, Weinmann O, Schwab ME (2004) The injured spinal cord spontaneously forms a new intraspinal circuit in adult rats. Nat Neurosci 7:269-277.

Canedo A, Lamas JA (1993) Pyramidal and corticospinal synaptic effects over reticulospinal neurones in the cat. J Physiol (Lond) 463:475-489. 
Chen R, Cohen LG, Hallett M (1997) Role of the ipsilateral motor cortex in voluntary movement. Can J Neurol Sci 24:284-291.

Darian-Smith I, Galea MP, Darian-Smith C (1996) Manual dexterity: how does the cerebral cortex contribute? Clin Exp Pharmacol Physiol 23:948-956.

Davies HE, Edgley SA (1994) Inputs to group II-activated midlumbar interneurones from descending motor pathways in the cat. J Physiol (Lond) 479:463-473.

Edgley SA, Jankowska E, Krutki P, Hammar I (2003) Both dorsal horn and lamina VIII interneurones contribute to crossed reflexes from group II muscle afferents. J Physiol (Lond) 552:961-974.

Feydy A, Carlier R, Roby-Brami A, Bussel B, Cazalis F, Pierot L, Burnod Y, Maier MA (2002) Longitudinal study of motor recovery after stroke: recruitment and focusing of brain activation. Stroke 33:1610-1617.

Floeter MK, Sholomenko GN, Gossard JP, Burke RE (1993) Disynaptic excitation from the medial longitudinal fasciculus to lumbosacral motoneurons: modulation by repetitive activation, descending pathways, and locomotion. Exp Brain Res 92:407-419.

Galea MP, Darian-Smith I (1997) Corticospinal projection patterns following unilateral section of the cervical spinal cord in the newborn and juvenile macaque monkey. J Comp Neurol 381:282-306.

Gossard JP, Floeter MK, Degtyarenko AM, Simon ES, Burke RE (1996) Disynaptic vestibulospinal and reticulospinal excitation in cat lumbosacral motoneurons: modulation during fictive locomotion. Exp Brain Res 109:277-288.

Grillner S, Hongo T, Lund S (1968) Reciprocal effects between two descending bulbospinal systems with monosynaptic connections to spinal motoneurones. Brain Res 10:477-480.

Hammar I, Bannatyne BA, Maxwell DJ, Edgley SA, Jankowska E (2004) The actions of monoamines and distribution of noradrenergic and serotoninergic contacts on different subpopulations of commissural interneurons in the cat spinal cord. Eur J Neurosci 19:1305-1316.

He XW, Wu CP (1985) Connections between pericruciate cortex and the medullary reticulospinal neurons in cat: an electrophysiological study. Exp Brain Res 61:109-116.

Hongo T, Jankowska E, Lundberg A (1969) The rubrospinal tract. II. Facilitation of interneuronal transmission in reflex paths to motoneurones. Exp Brain Res 7:365-391.

Illert M, Lundberg A, Tanaka R (1975) Integration in a disynaptic corticomotoneuronal pathway to the forelimb in the cat. Brain Res 93:525-529.

Illert M, Lundberg A, Tanaka R (1977) Integration in descending motor pathways controlling the forelimb in the cat. 3. Convergence on propriospinal neurones transmitting disynaptic excitation from the corticospinal tract and other descending tracts. Exp Brain Res 29:323-346.

Illert M, Jankowska E, Lundberg A, Odutola A (1981) Integration in descending motor pathways controlling the forelimb in the cat. 7. Effects from the reticular formation on $\mathrm{C} 3-\mathrm{C} 4$ propriospinal neurones. Exp Brain Res 42:269-281.

Ito K, McCarley RW (1987) Physiological studies of brainstem reticular connectivity. I. Responses of mPRF neurons to stimulation of bulbar reticular formation. Brain Res 409:97-110.

Jankowska E, Hammar I, Slawinska U, Maleszak K, Edgley SA (2003) Neuronal basis of crossed actions from the reticular formation upon feline hindlimb motoneurons. J Neurosci 23:1867-1878.

Kably B, Drew T (1998) Corticoreticular pathways in the cat. I. Projection patterns and collaterization. J Neurophysiol 80:389-405.

Keizer K, Kuypers HG (1984) Distribution of corticospinal neurons with collaterals to lower brain stem reticular formation in cat. Exp Brain Res 54:107-120.

Keizer K, Kuypers HG (1989) Distribution of corticospinal neurons with collaterals to the lower brain stem reticular formation in monkey (Macaca fascicularis). Exp Brain Res 74:311-318.

Kucera P, Wiesendanger M (1985) Do ipsilateral corticospinal fibers participate in the functional recovery following unilateral pyramidal lesions in monkeys? Brain Res 348:297-303.

Kuypers HG, Lawrence DG (1967) Cortical projections to the red nucleus and the brain stem in the Rhesus monkey. Brain Res 4:151-188.
Lacroix S, Havton LA, McKay H, Yang H, Brant A, Roberts J, Tuszynski MH (2004) Bilateral corticospinal projections arise from each motor cortex in the macaque monkey: a quantitative study. J Comp Neurol 473:147-161.

Lawrence DG, Kuypers HG (1968) The functional organization of the motor system in the monkey. I. The effects of bilateral pyramidal lesions. Brain 91:1-14.

Li Q, Martin JH (2000) Postnatal development of differential projections from the caudal and rostral motor cortex subregions. Exp Brain Res 134:187-198.

Liu Y, Rouiller EM (1999) Mechanisms of recovery of dexterity following unilateral lesion of the sensorimotor cortex in adult monkeys. Exp Brain Res 128:149-159.

Lundberg A (1979) Integration in propriospinal motor centre controlling the forelimb in the cat. In: Integration in the nervous system (Asanuma H, Wilson VS, eds), pp 47-65. Tokyo: Igaru-Shoin.

Matsuyama K, Drew T (1997) Organization of the projections from the pericruciate cortex to the pontomedullary brainstem of the cat: a study using the anterograde tracer Phaseolus vulgaris-leucoagglutinin. J Comp Neurol 389:617-641.

Matsuyama K, Jankowska E (2004) Coupling between feline cerebellum (fastigial neurons) and motoneurons innervating hindlimb muscles. J Neurophysiol 91:1183-1192.

Matsuyama K, Ohta Y, Mori S (1988) Ascending and descending projections of the nucleus reticularis gigantocellularis in the cat demonstrated by the anterograde neural tracer, Phaseolus vulgaris leucoagglutinin (PHA-L). Brain Res 460:124-141.

Matsuyama K, Takakusaki K, Nakajima K, Mori S (1997) Multi-segmental innervation of single pontine reticulospinal axons in the cervico-thoracic region of the cat: anterograde PHA-L tracing study. J Comp Neurol 377:234-250.

Matsuyama K, Kobayashi Y, Takakusaki K, Mori S, Kimura H (1993) Termination mode and branching patterns of reticuloreticular and reticulospinal fibers of the nucleus reticularis pontis oralis in the cat: an anterograde PHA-L tracing study. Neurosci Res 17:9-21.

McCarley RW, Ito K, Rodrigo-Angulo ML (1987) Physiological studies of brainstem reticular connectivity. II. Responses of mPRF neurons to stimulation of mesencephalic and contralateral pontine reticular formation. Brain Res 409:111-127.

Nyberg-Hansen R, Mascitti TA (1964) Sites and mode of termination of fibers of the vestibulospinal tract in the cat. J Comp Neurol 122:369-387.

Pettersson LG, Blagovechtchenski E, Perfiliev S, Krasnochokova E, Lundberg A (2000) Recovery of food-taking in cats after lesions of the corticospinal (complete) and rubrospinal (complete and incomplete) tracts. Neurosci Res 38:109-112.

Porter R, Lemon RN (1993) Corticospinal function and voluntary movement, Chap 2-4. Oxford: Clarendon.

Strens LH, Fogelson N, Shanahan P, Rothwell JC, Brown P (2003) The ipsilateral human motor cortex can functionally compensate for acute contralateral motor cortex dysfunction. Curr Biol 13:1201-1205.

Takakusaki K, Ohta Y, Mori S (1989) Single medullary reticulospinal neurons exert postsynaptic inhibitory effects via inhibitory interneurons upon alpha-motoneurons innervating cat hindlimb muscles. Exp Brain Res 74:11-23.

Ugolini G, Kuypers HG (1986) Collaterals of corticospinal and pyramidal fibres to the pontine grey demonstrated by a new application of the fluorescent fibre labeling technique. Brain Res 365:211-227.

Weiller C, Chollet F, Friston KJ, Wise RJ, Frackowiak RS (1992) Functional reorganization of the brain in recovery from striatocapsular infarction in man. Ann Neurol 31:463-472.

Whishaw IQ, Gorny B, Sarna J (1998) Paw and limb use in skilled and spontaneous reaching after pyramidal tract, red nucleus and combined lesions in the rat: behavioral and anatomical dissociations. Behav Brain Res 93:167-183.

Wilson VJ, Yoshida M (1969) Comparison of effects of stimulation of Deiters' nucleus and medial longitudinal fasciculus on neck, forelimb, and hindlimb motoneurons. J Neurophysiol 32:743-758. 